\title{
A Probability-Based Prediction Framework for Stress Identification
}

\author{
Dong-soo Han, Jisoo Song, Janarbek Matai, Minkyu Lee \\ School of Engineering, \\ Information and Communications University \\ P.O.Box77, Yusong, Daejeon 305-600, Korea \\ \{dshan, bulldoger, janarbek, niklaus\}@icu.ac.kr
}

\begin{abstract}
In this paper, we propose a probability-based prediction framework for stress identification. The framework predicts the stress index of someone using vital signals and symptoms. The framework largely consists of learning stage and prediction stage. In learning stage, once vital signals and symptoms of stress group and normal group are available individually, a feature space is constructed. Vital signals and symptoms gathered from pre-identified subjects form the feature space. The framework constructs a matrix for each group which holding appearance frequencies of vital signals and symptom pair that is found in the feature space. Another matrix, SCAP matrix, is constructed using two matrices. It contains probabilities that indicate a certain vital signals-symptom pair may be found in stress group and the framework prepares weight vectors in order to direct the use of vital signals and symptoms in personalized manner. In prediction stage, a probability equation is devised based on SCAP matrix and the weight vectors. Once the vital signal and symptom pair of someone is available, the probability that indicates a person may belong to a stress group is computed using the probability equation. In this paper, we conduct a simple validation test for this framework using vital signal and symptom data obtained from 29 subjects. The method is revealed to have high potential to improve probability of stress identification further after more learning data is accumulated.
\end{abstract}

\section{Introduction}

Stress is a pressure from outside, and people under stress feel tense internally. Stress can be classified into acute stress and chronic stress based on style. Acute stress comes from specific situation like a heavy work and diseases. There are some researches to identify acute stress because the cause of acute stress is obvious. However, it is non-trivial to identify chronic stress. Stress is not always harmful. Sometimes suitable stress can be a motivator for the creativity of people. But excessive stress can bring serious medical problems. Thus stress identification has long been an issue not only to patients and doctors but also to ordinary people. However, there is no concrete method to predict the stress index of a subject yet. This is because many vital signals and symptoms are entangled with stress but their relation is not clearly understood yet.

Several vital signals are known to have correlations with stress. For example, Heart Rate Variability (HRV), body temperature, blood sugar level, body fat ratio, and cortisol hormone are reported to show significant or subtle differences in stress group $[1,2$, 3]. In fields, a checklist which checks symptoms or causes of stress is usually used in a form of questionnaire to identify stress with the vital signal information. However, there are just a few studies that handle vital signals in integrated manner. Integrated information of vital signals and symptoms gathered from a stress group may be used for future reference and diagnosis. However, most conventional stress identification methods usually handle and interpret each vital signal in separate manner.

In this paper, we develop a probability based prediction framework for stress identification. It provides mechanisms to predict probabilities that indicate a subject may belong to a stress group using vital signal and symptom of stress group and normal group. This framework is distinguished from the conventional stress identification methods in that all the vital signals are handled in integrated manner within the framework. Furthermore, the vital signal and symptom data gathered in prediction stage can be used as feedback data for improving the framework.

The framework largely consists of learning stage and prediction stage. In learning stage, a feature space is constructed. Vital signals and symptoms gathered 
from pre-identified subjects form the feature space. The framework constructs a matrix for each group. The matrices hold appearance frequencies of vital signals and symptom pair that are found in the feature space.

Stress Combination Appearance Probability (SCAP) matrix is constructed using above two matrices. It contains probabilities that indicate a certain vital signals-symptom pair may be found in stress group and it plays as an important role in the prediction stage. Then we prepare a weight vector.

In prediction stage, a probability equation is devised based on SCAP matrix and the weight vector. Once the vital signal and symptom data of a subject are available, we can compute the probability that indicates the subject may belong to a stress group using the probability equation.

A simple validation for the framework was performed using vital signal and symptom data that are obtained from 29 subjects. It is revealed that the accuracy of prediction reached around $70 \%$. The accuracy of prediction will be improved further as the number of available vital signal and symptom data increases.

This paper is organized as follows, In Section 2, this paper briefly introduce traditional approaches on stress identification. In Section 3, this paper explains the details of the proposed framework in a step by step. In Section 4, the validation of the framework follows. Finally, this paper concludes in Section 5.

\section{Related Work}

There are many researches to predict the stress index of subject using vital signals. Most of them tried to reveal the change of four vital signals when subjects are under stress. They are Heart Rate Variability (HRV), body temperature, blood sugar level, and body fat ratio.

Among four vital signals, HRV is the most popularly used in stress identification. Nowadays, most hospitals use HRV in order to check someone's stress index. It is usually calculated by analyzing the time series of beat-to-beat intervals, in milliseconds. HRV decreases with age and generally, healthier people have lower HRV. In addition, people under stress have higher HRV because of meeting increased for oxygen and nutrients carried in the blood [1] [5]. For example, it is reported that Beats per Minutes of resting group are in the range of 60 to 90 and BPM of stress group is in the range of $200 \sim 220$.

Tim Lowenstein suggested a method for stress identification by comparing hand temperature with neck temperature [2]. The method is relatively easy and simple. According to the method, the temperature gap between hand and neck becomes the primary criterion for stress identification. If the hand temperature is much colder than neck temperature of a person, that person may have stress. This method is still in use in many fields.

Stress affects the secretion of insulin in a body. When a person is under stress, the body of the person produces more epinephrine hormone than usual. Epinephrine is known as adrenaline. Epinephrine enables people to use more glucose and fat to give the body extra energy so that it can respond promptly to the stressful situation. That is, epinephrine causes the release of stored glucose into the blood, and therefore increases blood glucose levels [5]. It is reported that blood sugar level is 4 to $7 \mathrm{mmol} / 1$ before meals, less than $10 \mathrm{mmol} / 190$ minutes after a meal, around $8 \mathrm{mmol} / \mathrm{l}$ at bedtime (or 7 to $10 \mathrm{mmol} / 1$ ) [3]. Thus we can identify stress by measuring the change of blood sugar level and comparing it with normal values.

It is also known that stress prompts the release of the cortisol hormone. This hormone breaks down muscle proteins and inhibits fat burning while causing fat deposition. Therefore, chronic stress may bring weight gain eventually.

However, there are several drawbacks in conventional approach that uses vital signals in a separate manner. First, the range of each signal is very dependent on individuals. That means a criterion for stress identification cannot be uniformly applied for every people. Second, since stress causes the change of multiple vital signals, stress identification must use multiple vital signals not just one single vital signal. It may be not sufficient to get a reliable result. Third, the correlations between stress and vital signals are not clear in some cases. For example, the correlation with stress and cortisol level is still questionable.

People can easily check their own stress index using the checklist. There are proven checklists that are effective in stress identification. But, one problem of checklist approach is that too many symptoms and questions should be correctly answered by people. Thus in some situations like mobile environment, the checklist approach is not adequate. Very simplified version of the checklist is necessary.

\section{A Probabilistic Framework}

\subsection{Preliminaries}

This paper assumes that stress causes the change of vital signals like HRV, temperature, blood sugar level, and body fat ratio and there is interrelation between the vital signals and symptoms like headache, dyspepsia, 
sensitive skin, feeling oppression in the chest, nervousness, forgetfulness, etc.

Thus if the appearance frequencies of vital signals and symptom pair are clearly perceived, we will be able to identify stress from appearance frequencies That means that if the appearance frequencies in a stress group appear differently from those in a normal group, then we can identify stress by analyzing the appearance frequencies of vital signals and symptom pair.

\subsection{The Big Picture}

The proposed framework largely consists of two stages: learning stage and prediction stage. Figure 1 shows the schematic view of the system.

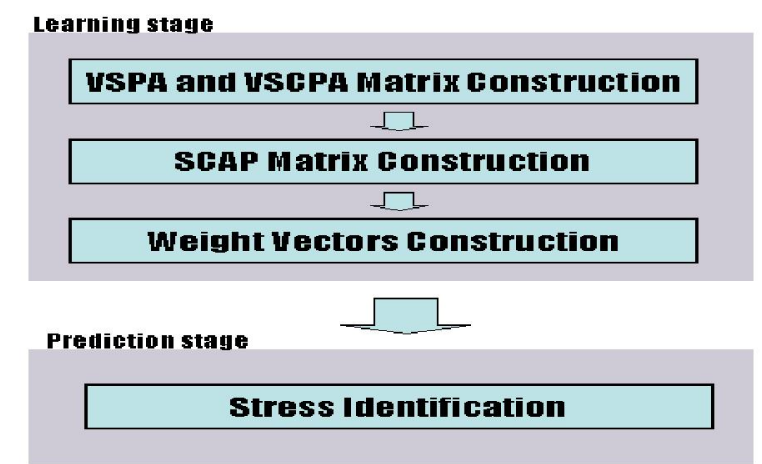

Fig. 1. The schematic view of the prediction system

In the learning stage, appearance frequencies of vital signals and symptom pair in a normal group and a stress group are registered in two matrices respectively. Sufficient number of vital signal and symptom data is essential for the successful learning. In the prediction stage, a probability equation is prepared in order to identify stress of an unknown subject using the vital signals and symptoms.

The learning stage is divided into three steps. In the first step, the appearance frequencies of vital signal and physical symptom in a normal group and stress group are counted and registered in two Vital sign-Symptom Pair Appearance (VSPA) matrices; one for normal group and another for stress group.

In the second step, Stress Combination Appearance (SCAP) matrix is constructed using above two VSCPA matrices. Each element of SCAP matrix represents the appearance probability of a certain vital signal and symptom pair in stress group.

The degree of influence of each vital signal and symptom pair to stress for each individual is different and the framework must give more weight to some vital signals. The method of giving a weight to something is usually used in the machine learning. Therefore, in the third step, weight vectors are prepared in order to direct the use of vital signals and symptoms in personalized manner.

In prediction state, a probability equation is devised based on SCAP matrix and weight vectors. The probability equation computes the probability with given vital signals and symptoms from a subject. In the following sections we explain the details of the each step.

\subsection{Learning Stage}

Stress Combination Appearance Probability (SCAP) matrix is the final result of the learning stage. SCAP matrix contains probability that a certain vital signal and symptom pair appears in stress group. Vital sign-Symptom Pair Appearance (VSPA) and Vital sign-Symptom Combination Pair Appearance (VSCPA) matrices are constructed for the construction of SCAP matrix.

\subsubsection{Vital sign-Symptom Pair Appearance (VSPA) Matrix.}

This paper explains VSPA matrix to help the understanding of VSPA matrix. Thus VSPA matrix needs not to be created in practice. In VSPA matrix, the column elements represent vital signals, and the row elements represent symptoms. When there are $n$ different vital signals $\left\{\mathrm{b}_{1}, \mathrm{~b}_{2}, \ldots, \mathrm{b}_{\mathrm{n}}\right\}$, and each vital signal $b_{i}$ is categorized into $k_{i}$ different types of signals, then

$$
\left\{b_{11}, b_{12}, \ldots, b_{1 k_{1}}, b_{21}, b_{22}, \ldots, b_{2 k_{2}}, \ldots, b_{n 1}, b_{n 2}, \ldots, b_{n k_{n}}\right\}
$$

constitute the column elements of VSPA matrix. In the same way, when there are $\mathrm{m}$ different physical symptoms $\left\{\mathrm{s}_{1}, \mathrm{~s}_{2}, \ldots, \mathrm{s}_{\mathrm{m}}\right\}$ and each symptom $\mathrm{s}_{\mathrm{i}}$ is categorized into $1_{1}$ different types of symptoms, then

$$
\left\{s_{11}, s_{12}, \ldots, s_{1 k_{1}}, s_{21}, s_{22}, \ldots, s_{2 k_{2}}, \ldots, s_{n 1}, s_{n 2}, \ldots, s_{n k_{n}}\right\}
$$

constitute the row elements of VSPA matrix. As a result, the size of VSPA matrix for above example becomes,

$$
\sum_{i=1}^{n} k_{i} \times \sum_{j=1}^{m} l_{j}
$$

Thus, it is simple and straightforward to construct a VSPA matrix once vital signal and symptom data is available. We suppose that there are two vital signals $\left\{\mathrm{b}_{1}, \mathrm{~b}_{2}\right\}$, and two physical symptoms $\left\{\mathrm{s}_{1}, \mathrm{~s}_{2}\right\}$. In addition, each vital signal and physical symptom has two different types of vital signals and physical symptoms respectively. Then, $\left\{b_{11}, b_{12}, b_{21}, b_{22}\right\}$ and $\left\{\mathrm{s}_{11}, \mathrm{~s}_{12}, \mathrm{~s}_{21}, \mathrm{~s}_{22}\right\}$ constitute column and row elements 
of VSPA matrix respectively. Consequently, $4 \times 4$ VSPA matrix is constructed in this case.

Suppose further that members $\left\{\mathrm{p}_{1}, \mathrm{p}_{2}, \mathrm{p}_{3}\right\}$ are in a stress group and members $\left\{\mathrm{p}_{4}, \mathrm{p}_{5}, \mathrm{p}_{6}\right\}$ are in a normal group Also, suppose that the members have the following < vital-signal, symptom> pairs: member of stress group have

Pair $\left(\mathrm{p}_{1}\right)=\left\{\left\langle\mathrm{b}_{11}, \mathrm{~s}_{11}>,<\mathbf{b}_{11}, \mathrm{~s}_{22}>,\left\langle\mathrm{b}_{22}, \mathrm{~s}_{11}\right\rangle,\left\langle\mathrm{b}_{22}, \mathrm{~s}_{22}>\right\}\right.\right.$ Pair $\left(\mathrm{p}_{2}\right)=\left\{\left\langle\mathrm{b}_{11}, \mathrm{~s}_{11}>,<\mathrm{b}_{11}, \mathrm{~s}_{21}>,<\mathrm{b}_{21}, \mathrm{~s}_{11}>,<\mathrm{b}_{21}, \mathrm{~s}_{21}>\right\}\right.$ Pair $\left(\mathrm{p}_{3}\right)=\left\{\left\langle\mathrm{b}_{12}, \mathrm{~s}_{12}>,\left\langle\mathrm{b}_{12}, \mathrm{~s}_{22}\right\rangle,\left\langle\mathrm{b}_{22}, \mathrm{~s}_{12}\right\rangle,\left\langle\mathrm{b}_{22}, \mathrm{~s}_{22}\right\rangle\right\}\right.$ and, member of normal group have

Pair $\left(\mathrm{p}_{4}\right)=\left\{\left\langle\mathrm{b}_{12}, \mathrm{~s}_{12}>,\left\langle\mathrm{b}_{12}, \mathrm{~s}_{21}\right\rangle,\left\langle\mathrm{b}_{21}, \mathrm{~s}_{12}>,<\mathrm{b}_{21}, \mathrm{~s}_{21}\right\rangle\right\}\right.$ Pair $\left(\mathrm{p}_{5}\right)=\left\{\left\langle\mathrm{b}_{12}, \mathrm{~s}_{12}>,<\mathrm{b}_{12}, \mathrm{~s}_{21}>,<\mathrm{b}_{22}, \mathrm{~s}_{12}>,<\mathrm{b}_{22}, \mathrm{~s}_{21}>\right\}\right.$ Pair $\left(\mathrm{p}_{6}\right)=\left\{\left\langle\mathrm{b}_{11}, \mathrm{~s}_{11}>,\left\langle\mathrm{b}_{11}, \mathrm{~s}_{22}>,\left\langle\mathrm{b}_{21}, \mathrm{~s}_{11}\right\rangle,\left\langle\mathrm{b}_{21}, \mathrm{~s}_{22}>\right\}\right.\right.\right.$ respectively.

Element $\operatorname{VSPA}(\mathrm{K})_{\mathrm{ij}}$ represents vital signal and symptom pair $\left\langle b_{i}, s_{j}\right\rangle$ in member of group $K$ and its value is decided by counting the appearance frequencies of the pair in group $\mathrm{K}$. $\mathrm{K}$ denotes either normal or stress group. Fig. 2 shows the VSPA matrices obtained from the example cases.
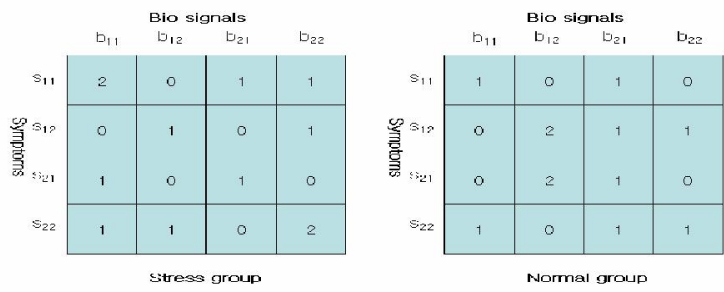

Fig. 2. VSPA(stress) and VSPA(normal) matrices

\subsubsection{Vital sign-Symptom Pair Combination Appearance (VSCPA) Matrix.}

VSPA matrix certainly provides a way of registering vital signal and symptom pair of a normal group and a stress group. However, we may miss some pairs when storing vital signal and symptom pair data in VSPA matrix because each vital signal and symptom is treated independently. We can easily consider that a vital signal may affect to other vital signals and a symptom may affect to other symptoms. Therefore, the VSPA matrix must be appropriately extended to accommodate this.

This paper introduces the notion of vital signal combination and symptom combination for this. Vital signal combination is a set of vital signals and symptom combination is a set of symptoms. Vital signSymptom Combination Pair Appearance (VSCPA) matrix is constructed by adding these vital signal combinations and symptom combinations to column and row of VSPA matrix.
In the example case, $\left\{\mathrm{b}_{11}, \mathrm{~b}_{21}\right\},\left\{\mathrm{b}_{11}, \mathrm{~b}_{22}\right\},\left\{\mathbf{b}_{12}, \mathrm{~b}_{21}\right\}$, $\left\{b_{12}, b_{22}\right\}$ are added to column elements, and symptom combinations, $\left\{\mathrm{s}_{11}, \mathrm{~s}_{21}\right\},\left\{\mathrm{s}_{11}, \mathrm{~s}_{22}\right\},\left\{\mathrm{s}_{12}, \mathrm{~s}_{21}\right\},\left\{\mathrm{s}_{12}, \mathrm{~s}_{22}\right\}$ are added to row elements. The value of each element is computed in the same manner as used in VSPA matrix. Consequently, $8 \times 8$ VSCPA matrix is constructed in this case. Fig. 3 shows VSCPA matrix obtained form the example.
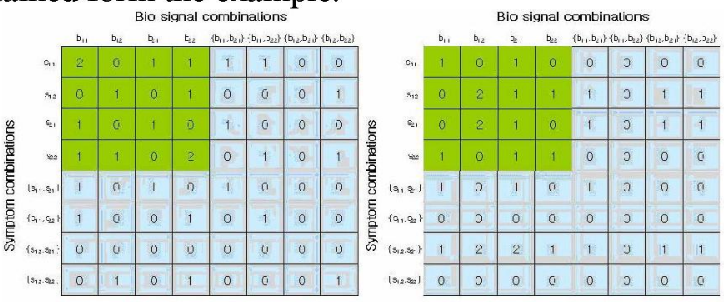

Siess aroud

Norral group

Fig. 3. VSCPA matrices for example stress and normal groups

\subsubsection{Stress Combination Appearance Probability (SCAP) Matrix.}

Once the above two VSCPA matrices are constructed, we can build a SCAP matrix. Each element of SCAP matrix contains the probability that a certain vital signal and symptom pair appears in stress group (more precisely, vital signal combination and symptom combination pair). The value of an element of SCAP matrix is computed by following equation:

$$
\mathrm{SCAP}_{\mathrm{ij}}=\frac{V S C P A(\text { Stress }) i j}{V S C P A(\text { Stress }) i j+V S C P A(\text { Normal }) i j}
$$

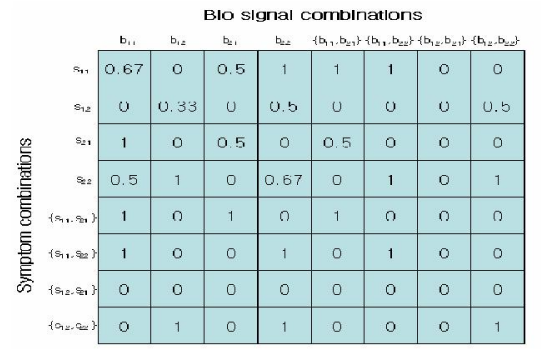

Fig. 4. SCAP matrix obtained from VSCPA matrices in Fig. 3.

\subsubsection{Weight vector.}

As mentioned above, it may be proper that giving more weight to several vital signals and symptoms than others. Giving weights to something is typically used in machine learning. However, SCAP matrix doesn't contain weight value. This paper introduces two weight vectors in order to give a different weight to each vital signal and symptom.

The two weight vectors are one for vital signals, 
Weight $_{\text {vital_signal }}=\left\{\mathrm{wv}_{1}, \mathrm{wV}_{2}, \ldots, \mathrm{wV}_{\mathrm{n}}\right\}$ and another for symptoms,

$$
\text { Weight }_{\text {symptom }}=\left\{\mathrm{ws}_{1}, \mathrm{ws}_{2}, \ldots, \mathrm{ws}_{\mathrm{m}}\right\}
$$

respectively. Here, $\sum w b=1, \sum w s=1$.

This paper assumes that the initial values of weight vectors are given by experts. It's also possible method that finds optimal values using a separate way.

If a row or a column element represents a combination of vital signals or symptoms, then the average weight of the vital signals or symptoms in the combinations becomes the average value of corresponding row or column element. For example, there is a weight vector for two vital signals like Weight $_{\text {vital_signal }}=\{0.3,0.7\}$. Then the weight value of combination of two vital signals becomes $(0.3+0.7) /$ $2=0.5$.

We expect that weight vectors will work as a key role in order to serve people in a personalized manner. The individual management is very important in the some area like mobile environment.

\subsection{Prediction Stage}

In this section, the framework develops a probability equation which computes probabilities that indicate a subject may belong to a stress group. It's kind of a stress index. The probability equation is developed based on SCAP and weight vectors explained in previous sections. Therefore, suppose that an $\mathbf{p} \times \mathbf{q}$ SCAP matrix, and an two weight vectors have been prepared. Also, let $\mathrm{sc}\left(\mathrm{p}_{\mathrm{i}}\right)=\left\{\mathrm{sc}_{1}, \mathrm{sc}_{2}, \ldots, \mathrm{sc}_{\mathrm{m}}\right\}$ and be the set of symptom combinations detected by a person $\mathrm{p}_{\mathrm{i}}$, and let

$b c\left(s c_{j}\right)=\left\{b c_{j} x_{j}(1), b c_{j} x_{j}(2), \ldots, b c_{j} x_{j}(k)\right\}$

where, $1 \leq \mathrm{j} \leq \mathrm{p}$ and $1 \leq \mathrm{x}_{\mathrm{j}}(\mathrm{k}) \leq \mathrm{q}$

be the set of vital signal combinations detected by person $\mathrm{p}_{\mathrm{i}}$ in the row of $\mathrm{sc}_{\mathrm{j}}$ in SCAP and WA matrices. Note that, SCAP matrix and two weight vectors share exactly the same column and row elements.

Then, the probability equation that a person $p_{i}$ with a set of combination par $\mathrm{CP}\left(\mathrm{p}_{\mathrm{j}}\right)$ is defined by

$$
\begin{aligned}
& \mathrm{P}(\mathrm{p} \in \text { stress group } \mid \mathrm{p} \text { has } \mathrm{CP}(\mathrm{p}))= \\
& \sum_{u=1}^{n}\left[\frac{w \operatorname{eight}\left(s c_{u}\right)}{\sum_{v=1}^{n} w \operatorname{weight}\left(s c_{v}\right)} \cdot\left(\sum_{i=X_{u}(1)}^{x_{u}(k)} \frac{\left.w \operatorname{eight}\left(b c_{u}\right) \cdot S C A P s c_{u} b c_{u}\right)}{\sum_{j=x_{u}(1)}^{x_{u}(k)} w \operatorname{wetght}\left(b c_{u}\right)}\right]\right.
\end{aligned}
$$

Suppose that we apply the above equation to an example case $\mathrm{p}_{\mathrm{i}}$, whose $\mathrm{sc}\left(\mathrm{p}_{\mathrm{i}}\right)$ is $\left\{\left\{\mathrm{s}_{11}\right\},\left\{\mathrm{s}_{21}\right\} .\left\{\mathrm{s}_{11}, \mathrm{~s}_{21}\right\}\right\}$, and bc $\left(\left\{s_{11}\right\}\right), b c\left(\left\{s_{21}\right\}\right), b c\left(\left\{s_{11}, s_{21}\right\}\right)$ have the same set, $\left(\left\{b_{11}\right\},\left\{b_{21}\right\},\left\{b_{11}, b_{21}\right\}\right)$ as shown in the matrix in Fig.5.

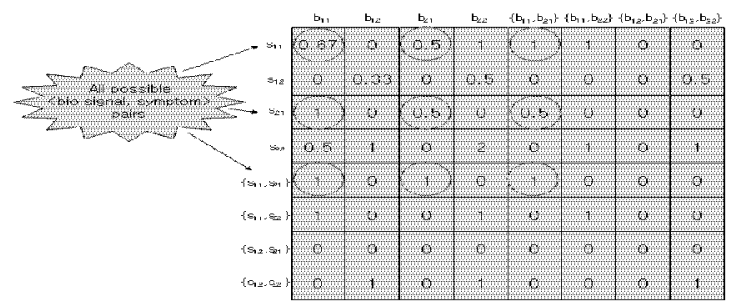

Fig.5. several values are selected in SCAP matrix

Since the values of weight $\left(\left\{\mathrm{s}_{11}\right\}\right)$, weight $\left(\left\{\mathrm{s}_{21}\right\}\right)$, and weight $\left(\left\{\mathrm{s}_{11}, \mathrm{~s}_{21}\right\}\right)$, are $0.6,0.4,0.5$ respectively, and the values of weight $\left(\left\{\mathrm{b}_{11}\right\}\right)$, weight $\left(\left\{\mathrm{b}_{21}\right\}\right)$, and weight $\left(\left\{b_{11}, b_{21}\right\}\right)$, are $0.3,0.7,0.5$ respectively, the probability is computed by:

$$
\begin{aligned}
\text { probability } & =\frac{0.6}{0.6+0.4+0.5} *\left(\frac{0.67 * 0.3}{0.3+0.7+0.5}+\frac{0.5 * 0.7}{0.3+0.7+0.5}+\frac{1 * 0.5}{0.3+0.7+0.5}\right) \\
& +\frac{0.4}{0.6+0.4+0.5} *\left(\frac{1 * 0.3}{0.3+0.7+0.5}+\frac{0.5 * 0.7}{0.3+0.7+0.5}+\frac{0.5 * 0.5}{0.3+0.7+0.5}\right) \\
& +\frac{0.5}{0.6+0.4+0.5} *\left(\frac{1 * 0.3}{0.3+0.7+0.5}+\frac{1 * 0.7}{0.3+0.7+0.5}+\frac{1 * 0.5}{0.3+0.7+0.5}\right) \\
& =0.772
\end{aligned}
$$

From this, we can conclude the person in this example is classified into stress group in $77.2 \%$ probability.

\section{Validation}

For the validation of the proposed framework, this paper captured 29 subjects' vital signals in two different conditions. One group of vital signals is captured when subjects are under acute stress and another is captured when subjects are in normal condition. Four kinds of vital signals such as, RR, HR, SDNN, and SI are extracted or analyzed from ECG signals of individual person. Here, RR, HR, SDNN, SI stand for R to R interval, Heart rate, Standard deviation of full electro cardiac signal, Stress index, respectively.

One problem we have in the validation is that the number of captured data is not sufficient for the validation of proposed method. The proposed method usually requires large number of data for learning. This paper increased the number of data using the captured real data in order to solve this problem. When a real data $x$ is given, the data $x$ is considered as a mean value and the distribution of the data is assumed to take normal distribution. Then once the variance of the distribution $N(x, \sigma)$ is decided, we can generate required number of data from $N(x, \sigma)$.

Another issue is to decide the number of types for each vital signal. The number of types is also arbitrarily decided. For example, we can use 3 different types for RR signals like Low, Medium, High, and give 
the range $[0,1]$ for Low type, [1, 2] for Medium type, and $[2, \infty]$ for High type.

The last issue we have to resolve for the validation is how to generate Weight vectors. In this validation, we assigned the same weight to each vital signal. The following is the summary of the conditions used for our validation.

- Each test repeated 20 times.

- Subjects in test set are chosen randomly among 29 subjects.

- Variance $=0.2$.

- Weight Vector $=\{0.25,0.25,0.25,0.25\}$.

- Number of vital signal types $=4$.

- Number of generated data from a real data $=5$.

Since the sensitivities and specificities are dependent on the cut-off probabilities used in identifying stress group, the change of sensitivities and specificities were measured for the cut-off probabilities from 0.2 to 0.7 .

As expected, the sensitivities decrease and specificities increase as we increase the cut-off value. $68.18 \%$ sensitivity and $69.6 \%$ specificity were achieved for cut-off probability 0.434 .

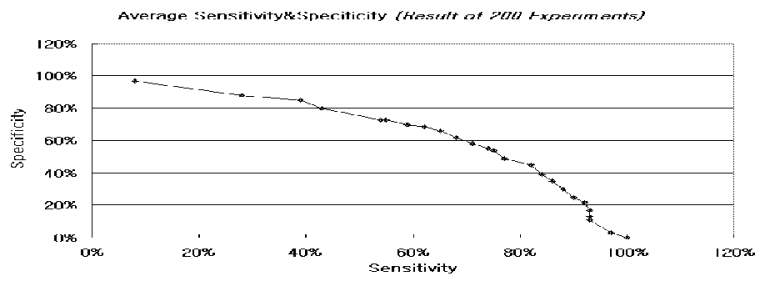

Fig.6. Average sensitivities and specificities depending in number of subjects in test set

The obtained sensitivities and specificities may or may not acceptable by users and situations. However we can draw several conclusions out of the results. First, the obtained sensitivities and specificities are superior to those of the result obtained using only a single vital signals. This indicates that it is effective to use combination information of vital signals in identifying stress group.

Second, there is still probability to improve the results. In this paper, we conduct validation under only one condition. This framework may have better results if we validate our method in different conditions.

Third, when the amount of learning data increases, the framework can have much more improved and get reliable result. In this validation, the symptom was not included because the number of data is not sufficient for the validation. Much more number of data is necessary for such validation. In future, we are planning to gather more data including symptom information.

\section{Conclusion}

In this paper, we have presented a probability-based prediction framework for stress identification using combination of vital signals and symptoms from two groups. This framework is different from conventional approach in that it uses both vital signals and symptoms in forming learning combinations for the framework.

This framework comprises two stages. In learning stage, the framework builds a SCAP matrix holding probabilities that a certain vital signal and symptom pair appears in stress group and constructs weight vectors in order to give more weight to some vital signals or symptoms for a personalized service. In prediction stage, the framework identifies stress group using a probability equation which is devised based on SCAP and two weight vectors.

Even in limited validation, the method is revealed to have high potential to improve its capability of identifying stress group further as more learning data is accumulated.

In future we are planning to adapt this framework to the mobile environment. In addition, after gathering more data of vital signals and symptoms, we expect the accuracy of validation would be improved further. We also believe that this approach can be used in other areas like diabetes and stock price prediction with slight modifications.

\section{References}

[1] Jan Nissl, RN, and BS, Pulse Management, Yahoo Encyclopedia, June 29, 2004.

[2] Tim Lowenstein, 10 Second STRESS TEST, http://www.cliving.org, 1997.

[3] Ian W Campbell and Patrick Davey, Blood glucose levels, http://www.netdoctor.co.uk/, August 24, 2004.

[4] Robert Nolan, Heart Rate Variability (HRV), Thought Technology Ltd. , 1996.

[5] Kattie Payne, Preventing blood sugar emergencies, Yahoo Encyclopedia, February 14, 2003.

[6] Ikemi A, Tomita S, and Hayashida Y, Thermographical analysis of the warmth of the hands during the practice of self-regulation method, Psychotherapy-and-Psychosomatics, 1988, Vol 50(1) 22-28. 